\title{
PRODUCCION HIDROPÓNICA DE CHILE HABANERO NEGRO (Capsicum chinense Jacq.)
}

\section{BLACK HABANERO PEPPER (Capsicum chinense Jaq.) HYDROPONICS PRODUCTION}

\section{Mario Tapia-Vargas ${ }^{1}$, Antonio Larios-Guzmán', Deisy D. Díaz-Sánchez², Gabriela Ramírez-Ojeda², Anselmo Hernández-Pérez ${ }^{1}$, Ignacio Vidales-Fernández ${ }^{1}$ y Héctor Guillén-Andrade ${ }^{3}$}

\begin{abstract}
'Campo Experimental Uruapan, Instituto Nacional de Investigaciones Forestales, Agrícolas y Pecuarias. Av. Latinoamericana 1101. 60080, Uruapan,

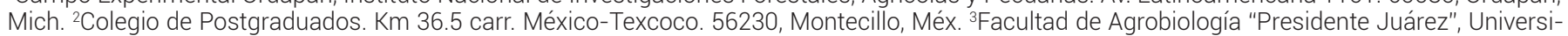
dad Michoacana de San Nicolás de Hidalgo. Paseo de la Revolución esq. Niza. 60160, Uruapan, Mich.
\end{abstract}

Autor de correspondencia (tapia.luismario@inifap.gob.mx)

\section{RESUMEN}

El chile habanero negro (Capsicum chinense Jacq.) es un cultivo que se produce en áreas reducidas de la costa michoacana, a pesar de que tiene importancia económica por su elevado grado de picor y demanda culinaria. Un factor que limita la producción en invernadero es la polinización y por consiguiente el rendimiento de fruto por lo que el objetivo de este trabajo fue evaluar el efecto de la aplicación de un complejo hormonal en la producción de fruto de chile habanero negro (CHN). El experimento se realizó en un invernadero de acrílico y plástico. El trasplante se efectuó en abril de 2015 en bolsas de polietileno negro con $4 \mathrm{~kg}$ de tezontle, y se aplicó diariamente una solución nutritiva balanceada por fertirriego (hasta $1.0 \mathrm{~L} \mathrm{~d}^{-1}$ ) con sistema de riego por goteo. Los tratamientos fueron 1) aplicación de complejo hormonal en base a citocininas $(\mathrm{CH})$ en dosis de $0.5 \mathrm{~mL} \mathrm{~L}^{-1}$ cada $10 \mathrm{~d}$, y 2) Testigo sin aplicación (T). El diseño experimental fue completamente al azar con 20 repeticiones, con cada planta como una repetición. Las variables evaluadas fueron altura de planta, grosor de tallo, longitud, diámetro y rendimiento de fruto, y concentración de capsaicinoides. Se aplicó análisis de varianza y se realizó comparación de medias con Tukey $(P<0.05)$. Los resultados indicaron que todas las variables evaluadas excepto diámetro de fruto, fueron estadísticamente significativas con valores más altos para el tratamiento $\mathrm{CH}$, sobre todo en rendimiento por planta (> $160 \%$ ), peso de fruto $(>30 \%)$ y vigor del cultivo (> 15\%). Asimismo, el contenido de alcaloides fue $60 \%$ más alto en $\mathrm{CH}$ que en T. La producción y calidad de fruto de $\mathrm{CHN}$ es factible de incrementar mediante la aplicación de $\mathrm{CH}$ formulado con una mezcla de citocininas.

Palabras clave: Capsicum chinense, capsaicinoides, calidad de fruto, invernadero.

\section{SUMMARY}

Black habanero pepper (Capsicum chinense Jacq.), is a crop grown in small areas of the Michoacan coast, despite its economic importance given by its high degree of pungency and high culinary demand. A factor that restrains greenhouse production is pollination and therefore fruit yield; thus, the aim of the study was to evaluate the effect of the application of a hormonal complex on black habanero pepper $(\mathrm{CNH})$ fruit production. The experiment was carried out under acrylic and plastic greenhouse. Transplanting was performed in April 2015 into black plastic bags filled with $4.0 \mathrm{~kg}$ of tezontle (red gravel), a balanced nutritive solution was applied on a daily basis through ferti-irrigation (up to 1.0 L per day) using a drip system. Treatments consisted of 1 ) hormonal complex based on cytokinins $(\mathrm{CH})$ at a dose of $0.5 \mathrm{~mL} \mathrm{~L}^{-1}$ applied every $10 \mathrm{~d}$, and 2) control without application (T). A completely randomized experimental design was used taking a single plant as a replication. The evaluated traits were plant height, stem width, fruit length, diameter and yield, and capsaicin content. Analysis of variance and Tukey multiple comparison test $(P<0.05)$ were applied using SAS for Windows. Results indicated that all evaluated traits, except for fruit diameter, were statistically significant with higher values for $\mathrm{CH}$ specially for yield per plant $(<160 \%)$, fruit weight $(>30 \%)$ and crop vigor (>15\%). Furthermore, alkaloid content was $60 \%$ higher in $\mathrm{CH}$ than in relation to $\mathrm{T}$. Fruit yield and quality of $\mathrm{CHN}$ is feasible to increase through cytokinins-based $\mathrm{CH}$ application.

Index words: Capsicum chinense, capsaicinoids, fruit quality, greenhouse.

\section{INTRODUCCIÓN}

El chile habanero (Capsicum chinense Jacq.), aunque no es originario de México, se ha apoderado del paladar del mexicano por su extraordinario picor: no se concibe la actividad culinaria nacional sin este condimento exclusivo de ciertos platillos y salsas. Su origen está en Sudamérica en la cuenca del Orinoco de donde se dispersó a las islas del Caribe en la época precolombina (González et al., 2006). De acuerdo con Uribe (2008), esta hortaliza fue traída de Cuba a Yucatán por los españoles, de allí su nombre "Habanero" y se considera uno de los chiles más picante del mundo con valores de 350 mil a 500 mil unidades Scoville.

Este tipo de hortaliza es muy especial y se cultiva principalmente en los tres estados de la península de Yucatán, región que obtuvo el certificado de Denominación de Origen (Borges-Gómez et al., 2014), en una superficie global de 990 ha (Ocampo et al., 2014), con rendimientos que pueden alcanzar de 10 a $14 \mathrm{t} \mathrm{ha}^{-1}$. En condiciones de invernadero la densidad de plantas es de 2.4 plantas $\mathrm{m}^{-2}$ y produce entre 7 y $12 \mathrm{~kg} \mathrm{~m}^{-2}$ de fruto comercial, lo que indica que cada planta rinde en promedio de 3 a $4.5 \mathrm{~kg}$ de fruto (Macías et al., 2013). 
La producción de chile en invernadero se efectúa principalmente para propiciar un mejor ambiente en el crecimiento y desarrollo de la planta, debido a que el viento, la radiación y la temperatura menor de $15^{\circ} \mathrm{C}$, pueden afectar el rendimiento y la calidad del fruto (Flores, 2013). Aún bajo estas condiciones, de acuerdo con Uribe (2008) los rendimientos son bajos por falta de tecnología de producción adecuada en relación con variedades, nutrición, prevención de enfermedades, etc.

En Michoacán el cultivo del chile habanero (llamado localmente "Manzano") se circunscribe a la zona de transición de Tacámbaro, mientras que el habanero negro se localiza en la costa michoacana: ambos tipos de chile se producen en huertos de superficies pequeñas, y su productividad es restringida por el clima y el manejo deficiente: sin embargo, el mercado es de alto potencial. El chile habanero negro es de muy alto picor y de precio elevado por lo que su cultivo en invernadero puede producir altos dividendos a los productores.

El chile habanero en invernadero incrementa el rendimiento de fruto (Cauich et al., 2006), pero se tienen factores limitantes como la polinización (Quezada-Euán, 2009), la cual puede ser propiciada por medios abióticos como el uso de hormonas y medios bióticos como el uso de insectos polinizadores (Dávila 2011). El uso de hormonas presenta ventajas, como mayor calidad de producto comercial, menor riesgo por picaduras de insectos polinizadores y mayor vigor del cultivo, pero puede interactuar con otros químicos foliares y causar estrés al cultivo. El objetivo de este trabajo fue evaluar el efecto de la aplicación de un complejo hormonal sobre la producción y desarrollo del cultivo del chile habanero negro bajo condiciones de invernadero.

\section{MATERIALES Y MÉTODOS}

El trabajo se realizó en un invernadero de acrílico y plástico calibre 600 ubicado en Uruapan Michoacán. De acuerdo con Gutiérrez-Contreras et al. (2010), el clima es del tipo $(A) C(m)\left(w_{2}\right)$, semicálido húmedo, el más húmedo de la zona de transición de la Sierra Purépecha. Se utilizó como contendor una bolsa de plástico negra calibre 600 de $5 \mathrm{~kg}$ de capacidad que se llenó con 4 kg de grava volcánica roja (tezontle) de 1 a $4 \mathrm{~cm}$ de diámetro y forma irregular.
El material vegetativo consistió de chile habanero negro originario de la costa michoacana, cuya semilla fue tratada con Captán Ultra ${ }^{\circledR}\left(1.0 \mathrm{~g} \mathrm{~L}^{-1}\right)$ asperjado y posteriormente la semilla se dejó secar a la sombra. La siembra se hizo en charolas de poliestireno en cavidades de $25 \mathrm{~cm}^{3}$ llenadas con turba rubia bien húmeda y drenada, cribada a $10 \mathrm{~mm}$, $60 \mathrm{~kg}$ de materia seca por $\mathrm{m}^{3}$ de turba, $\mathrm{pH}=4$ (medido en una mezcla 1:2 de suelo: agua), capacidad de intercambio de cationes (CIC) de 120 meq/100 g y c.a. $2 \%$ de cenizas.

La siembra se realizó en marzo de 2015 al depositar la semilla a $1 \mathrm{~mm}$ de profundidad y luego se tapó con plástico negro hasta la germinación, que ocurrió 15 d después. En abril se hizo el trasplante, cuando las plántulas tenían cuatro hojas verdaderas, y se colocó una planta por contenedor. A partir del trasplante hasta inicio de floración se aplicó la solución nutritiva para pimiento (Capsicum annuum L.) a 50 \% según recomendación de Andreau et al. (2015), y a partir de la floración y por resto del ciclo del cultivo se aplicó la solución nutritiva a $100 \%$, como se describe en el Cuadro 1.

El riego se aplicó diariamente a las 8:00 y a las 14:00 horas con goteros de $8 \mathrm{~L} \mathrm{~h}^{-1}$ y cuatro derivaciones por gotero, una hacia cada contenedor; por cada contenedor, desde el trasplante hasta inicio de floración se aplicó $0.5 \mathrm{~L} \mathrm{~d}^{-1}$, y de inicio de floración hasta el final del experimento se aplicó $1.0 \mathrm{~L} \mathrm{~d}^{-1}$. El agua de riego presentó un $\mathrm{pH}=6.3$ (evaluado con medidor de pH Horiba ${ }^{\circledR}$ Modelo B-213), salinidad de $0.154 \mathrm{mS} \mathrm{cm}^{-1}$ (evaluada con conductímetro Horiba ${ }^{\circledR}$ modelo B-173). La temperatura del agua fue de $18{ }^{\circ} \mathrm{C}$, proveniente del acuífero local. Por sus características químicas, el agua no presenta problemas para su utilización en la solución nutritiva (Carbone, 2015).

Los tratamientos fueron los siguientes: 1) Aplicación de compuesto hormonal a partir de inicio de botón floral hasta el final del experimento cada $10 \mathrm{~d}$ en dosis de 0.5 $\mathrm{mL} \mathrm{L^{-1 }}$ de agua en aplicación foliar sin surfactante, hasta cubrir el follaje, del producto comercial Formax-F® (compuesto hormonal constituido por un complejo de citocininas derivadas de adenina a una concentración de 14,000 ppm, que actúan como catalizadores de la diferenciación celular, inhiben el excesivo crecimiento vegetal y propician la inducción floral y mayor vigor del crecimiento de fruto); y 2) Testigo sin aplicación.

Cuadro 1. Solución nutritiva aplicada al chile habanero en invernadero (Andreau et al., 2015).

\begin{tabular}{|c|c|c|c|c|c|c|c|c|c|c|}
\hline \multicolumn{6}{|c|}{ Meq L-1 } & \multicolumn{5}{|c|}{$\mathrm{mg} \mathrm{L}^{-1}$} \\
\hline $\mathrm{NO}_{3}^{-}$ & $\mathrm{PO}_{4} \mathrm{H}_{2}^{-}$ & $\mathrm{K}^{+}$ & $\mathrm{Ca}^{++}$ & $\mathrm{Mg}^{++}$ & $\mathrm{SO}_{4}=$ & $\mathrm{Fe}^{++}$ & $\mathrm{Mn}^{++}$ & $\mathrm{Cu}^{++}$ & $\mathrm{Zn}^{++}$ & B \\
\hline 11.8 & 1.3 & 6.0 & 3.8 & 1.3 & 1.3 & 0.56 & 0.56 & 0.03 & 0.26 & 0.22 \\
\hline
\end{tabular}


Se utilizó un diseño experimental completamente al azar con 20 repeticiones, en donde cada planta fue una repetición. Los datos de altura de planta $(m)$ fueron registrados desde la base del tallo hasta el punto de crecimiento de la planta y los de grosor de tallo $(\mathrm{mm})$ a $5 \mathrm{~cm}$ de la base del tallo en forma semanal; rendimiento de frutos maduros de color oscuro (g/planta); los diámetros polar (mm) y ecuatorial $(\mathrm{mm})$ del fruto se midieron a la cosecha. El picor se midió con los contenidos de capsaicina y dihidrocapsaicina en extractos obtenidos de frutos completos, y las lecturas se hicieron en un cromatógrafo de líquidos de alta resolución Agilent modelo $1100 \circledR$ provisto con una columna Hypersil ODS MR ( $25 \mathrm{~cm}, 4.6 \mathrm{~mm}, 5 \mu \mathrm{M})$, fase móvil de gradiente constituido por acetonitrilo-agua a razón 45:55, con un flujo de $1.5 \mathrm{~mm} \mathrm{~min}^{-1}$ y duración de corrida de 20 min.

Previo a la extracción, los frutos fueron lavados con agua destilada, se disecaron y luego se liofilizaron en un equipo Labconco® (USA) en el cual se deshidrataron los frutos durante $4 \mathrm{~d}$. Las muestras deshidratadas se molieron hasta obtener un polvo fino. La extracción se realizó con $0.5 \mathrm{~g}$ de muestra y $5 \mathrm{~mL}$ de acetonitrilo en tubos Falcon de 15 $\mathrm{mL}$, los cuales fueron colocados a baño maría durante $5 \mathrm{~h}$ a $60{ }^{\circ} \mathrm{C}$ con agitación cada hora. Las muestras se dejaron enfriar a temperatura ambiente y en oscuridad.

El extracto resultante se filtró con acrodiscos de nailon ( $25 \mathrm{~mm}$ de diámetro, poro de $0.45 \mu \mathrm{m}$ ), enseguida se inyectaron $20 \mu \mathrm{L}$ por muestra en el cromatógrafo antes indicado. Para calcular el contenido de capsaicinoides se utilizaron estándares de capsaicina y dihidrocapsaicina. Los datos se analizaron estadísticamente con el programa SAS versión 2007 para Windows, con el procedimiento ANOVA y se efectuó la separación de medias con la prueba de Tukey a $5 \%$ de error.

\section{RESULTADOS Y DISCUSIÓN}

En el Cuadro 2 se presentan los resultados obtenidos en invernadero con respecto a rendimiento de fruto, peso medio del fruto, diámetro polar del fruto, diámetro ecuatorial del fruto, diámetro del tallo y altura de planta. Con excepción del diámetro ecuatorial del fruto, todas las variables fueron estadísticamente significativas entre tratamientos, donde la aplicación del complejo hormonal mostró un mejor desempeño en el vigor, crecimiento, tamaño del fruto y rendimiento de fruto por planta, superior en $133 \%$ al tratamiento testigo.

El mayor rendimiento de fruto comercial no sólo fue el resultado de un mayor número de frutos, sino que el peso medio de fruto también fue mayor en el tratamiento con hormonas ya que superó en $44 \%$ el peso medio alcanzado por el testigo. Esta misma tendencia se observa en diámetro polar que alcanza un tamaño mayor con una diferencia $19.1 \%$ en el tratamiento hormonal. En cuanto el vigor y crecimiento de la planta, también las diferencias son claramente significativas con un grosor del tallo superior en $20 \%$ en el tratamiento con hormonas en comparación con el testigo sin aplicación: esta relación es similar en la altura de planta con un incremento de $6.5 \%$ por efecto del tratamiento hormonal.

Los resultados obtenidos indican diferencias claras de una respuesta favorable de cultivo a la aplicación hormonal. El incremento en el crecimiento de la planta por la acción de varias hormonas del coctel hormonal se podría explicar por una mayor división y elongación celular y posteriormente en rápida diferenciación. Graillet et al. (2014) observaron que el rendimiento de fruto se incrementó por efecto de hormonas, ya que el testigo produjo sólo 286.8 g por planta, mientras que con aplicación de hormonas obtuvieron $1379 \mathrm{~g}$ por planta; los mayores rendimientos reportados por estos autores posiblemente tienen relación con el cultivar empleado pues fue un chile habanero amarillo el cual tiene mayor rendimiento y mayor tamaño que el habanero negro.

El incremento en más de $100 \%$ del rendimiento de fruto encontrado en este estudio (Cuadro 2) como producto de la aplicación del compuesto hormonal, presentó cierta semejanza con el encontrado por Ramírez-Luna et al. (2005), quienes reportaron $86 \%$ de aumento en el rendimiento y

Cuadro 2. Variables agronómicas de respuesta a la aplicación de complejo hormonal en chile habanero negro bajo invernadero.

\begin{tabular}{lcccccc}
\hline Tratamiento & $\begin{array}{c}\text { Rendimiento } \\
\text { de fruto } \\
\text { (g por planta) }\end{array}$ & $\begin{array}{c}\text { Peso medio del } \\
\text { fruto }(\mathrm{g})\end{array}$ & $\begin{array}{c}\text { Diámetro polar } \\
(\mathrm{mm})\end{array}$ & $\begin{array}{c}\text { Diámetro } \\
\text { ecuatorial } \\
(\mathrm{mm})\end{array}$ & $\begin{array}{c}\text { Grosor de } \\
\text { tallo }(\mathrm{mm})\end{array}$ & $\begin{array}{c}\text { Altura de } \\
\text { planta }(\mathrm{m})\end{array}$ \\
\hline Complejo hormonal & $418.9 \mathrm{a}$ & $29.9 \mathrm{a}$ & $29.2 \mathrm{a}$ & $24.4 \mathrm{a}$ & $16.1 \mathrm{a}$ & $1.46 \mathrm{a}$ \\
Testigo & $179.7 \mathrm{~b}$ & $20.7 \mathrm{~b}$ & $24.5 \mathrm{~b}$ & $22.7 \mathrm{a}$ & $13.4 \mathrm{~b}$ & $1.37 \mathrm{~b}$ \\
Tukey (DSH 0.05) & 70.2 & 4.9 & 4.1 & 2.9 & 1.3 & 0.04 \\
\hline
\end{tabular}

Promedios con la misma letra son iguales estadísticamente (Tukey, 0.05). 
mayor amarre de flores de chile habanero por el efecto de las citocininas. Rodríguez (2002) refirió un efecto similar con citocininas mediante las cuales obtuvieron mayor rendimiento y tamaño de fruto.

Las citocininas promueven mayor floración, cuajado de fruto y crecimiento (Ramírez et al., 2008). Un efecto importante de las citocininas es que demoran la senescencia foliar, incrementan la formación de yemas y brotes conducentes a flores y frutos e inducen mayor vigor y contenido de clorofila (Jordan y Casaretto, 2006), además, una mayor concentración de citocininas promueve una mayor translocación de fotosintatos en chile habanero (Ramírez et al., 2010). En este trabajo el complejo de citocininas le confirió al fruto un mayor tamaño (19 \%) y peso (44 \%) en comparación con el testigo (Cuadro 2).

En relación con el picor del fruto, los resultados se presentan en el Cuadro 3. La aplicación de citocininas produjo mayor contenido de alcaloides picantes en el fruto, ya que el tratamiento hormonal produjo un incremento de 57.8 $\%$ del alcaloide comparado con la concentración del fruto en el testigo; asimismo, para el chile habanero amarillo medido como referencia, el grado de picor fue $78.6 \%$ más bajo que el chile habanero negro tratado con el complejo hormonal, lo que indica claramente la superioridad en picor del chile habanero negro con respecto al habanero amarillo.

Cuadro 3. Contenido de alcaloide picante en chile habanero negro con aplicación de compuesto hormonal.

\begin{tabular}{lcc}
\hline Tratamiento & $\begin{array}{c}\text { Capsaicina } \\
\left(\mu \mathrm{g} \mathrm{g}^{-1}\right)\end{array}$ & $\begin{array}{c}\text { Dihidrocapsaicina } \\
\left(\mu \mathrm{g} \mathrm{g}^{-1}\right)\end{array}$ \\
\hline Complejo hormonal & $895.9 \mathrm{a}$ & $253.2 \mathrm{a}$ \\
Testigo & $567.7 \mathrm{~b}$ & $221.0 \mathrm{a}$ \\
$\begin{array}{l}\text { Habanero amarillo } \\
\text { (referencia) }\end{array}$ & $501.1 \mathrm{~b}$ & $244.5 \mathrm{a}$ \\
DSH (Tukey, 0.05) & 217.23 & 107.01 \\
\hline
\end{tabular}

$\mathrm{DSH}=$ diferencia significativa honesta. Promedios con la misma letra son estadísticamente iguales (Tukey, 0.05).

El nivel de picor del habanero negro también fue mayor en el tratamiento con hormonas. Ramírez et al. (2010), indican que la aplicación de compuestos hormonales incrementa el nivel de capsaicina en fruto en 20 a $40 \%$. En este trabajo el nivel de capsaicinas se incrementó un $37 \%$ en comparación con el testigo. El contenido de capsaicina puede considerarse bajo con relación a lo reportado por otros autores; Canto-Flick et al. (2008) reportaron más de $1000 \mathrm{\mu g} \mathrm{g}^{-1}$ de capsaicina, en cuya concentración la edad de la planta también juega un papel importante (MedinaLara et al., 2008), ya que los frutos presentan mayor poder irritante en los primeros cortes que en los últimos, de donde se tomó el fruto analizado en este trabajo.

\section{CONCLUSIONES}

Es factible incrementar la producción y calidad de fruto de CHN mediante la aplicación de compuestos hormonales basados en citocininas. El tratamiento hormonal presentó mayor rendimiento de fruto en $160 \%$, mayor peso de fruto, mayor longitud y mayor contenido del alcaloide en 40 \% en comparación con el testigo.

\section{BIBLIOGRAFÍA}

Andreau R., D. Giménez y J. Beltrano (2015) Soluciones nutritivas II. In: Cultivo en Hidroponía. J. Beltrano y D. O. Giménez (coords.) Facultad de Ciencias Agrarias y Forestales Universidad Nacional de La Plata. Buenos Aires, Argentina. pp:91-108.

Borges-Gómez L., C. Moo Kauil. J. Ruíz-Novelo. M. Osalde-Balam. C. González-Valencia, C. Yam-Chimal y F. Can Puc (2014) Suelos destinados a la producción de chile habanero en Yucatán: características físicas y químicas predominantes. Agrociencia 48:347-359.

Carbone A. (2015) Nutrición Mineral. In: Cultivo en Hidroponía. J. Beltrano y J. D. O. Giménez (coords.) Facultad de Ciencias Agrarias y Forestales Universidad Nacional de La Plata, Buenos Aires, Argentina. pp:61-72.

Canto-Flick, A., E. Balam-Uc, J. J. Bello-Bello, C. Lecona-Guzmán, D. SolísMarroquín, S. Avilés-Viñas, E. Gómez-Uc, G. López-Puc, N. SantanaBuzzy and L. G. Iglesias-Andreu (2008) Capsaicinoids content in habanero pepper (Capsicum chinense Jacq.): hottest known cultivars. HortScience 43:1344-1349.

Cauich O., J. J. G. Quezada E., V. Meléndez R., G. R. Valdovinos-Núñez and H. Moo-Valle (2006) Pollination of habanero pepper (Capsicum chinense) and production in enclosures using the stingless bee Nannotrigona perilampoides. Journal of Apicultural Research 45:125-130.

Gutiérrez-Contreras M., M. B. N. Lara-Chávez, H. Guillén-Andrade y A. T. Chávez-Bárcenas (2010) Agroecología de la franja aguacatera en Michoacán, México. Interciencia 35:647-653.

Dávila M. R. (2011) Polinización en invernadero. Hortalizas Junio 2011. http://www.hortalizas.com/horticultura-protegida/polinizacion-en-invernadero/ (Noviembre 2015)

Flores N. A. (2013) Invernaderos: el chile Habanero se abre mercado en diferentes países. Revista digital De Riego 11. http://editorialderiego.com/invernaderos-el-chile-habanero-se-abre-mercado-en-diferentes-pases/ (Noviembre 2015).

Graillet J. E. M., J. A. Hernández H., L. C. Alvarado G. y A. Retureta A. (2014) Evaluación de cuatro reguladores de crecimiento en chile habanero (Capsicum chinense Jacq) en Acayucan, Veracruz, México. Revista Biológica Agropecuaria Tuxpan 2:748-755

González E. T., L. Gutiérrez P. y F. Contreras M. (2006) El chile habanero de Yucatán. Revista Ciencia y Desarrollo 32:15-22.

Jordan M. y J. Casaretto (2006) Hormonas y reguladores del crecimiento: auxinas, giberelinas y citocininas. In: Fisiología Vegetal. F. A. Squeo y L. Cardemil (eds.). Ed. Universidad de La Serena. La Serena, Chile. pp:1-28.

Macías R.H., J. A. Muñoz V, M. A. Velásquez V., M. C. T Potisek T. y M. M. Villa C. (2013) Chile habanero: descripción de su cultivo en la península de Yucatán. Revista Chapingo Serie Zonas Áridas XII:37-43.

Medina-Lara F., I. Echevarría-Machado, R. Pacheco-Arjona., N. Ruiz-Lau, A. Guzmán-Antonio and M. Martínez-Estévez (2008) Influence of nitrogen and potassium fertilization on fruiting and capsaicin content in habanero pepper (Capsicum chinense Jacq.). Hortscience 43:1549-1554.

Ocampo T. P., J. M. Cocom V. y L. A. Puc C. (2014) Diagnóstico histórico de la producción de chile habanero, papaya, plátano y miel en el Sureste de México (Yucatán, Campeche, Quintana Roo, Chiapas, Tabasco y Veracruz). CICY A.C. Mérida, Yuc. México. 156 p. 
Quezada-Euán J. J. (2009) Potencial de las abejas nativas en la polinización de cultivos. Acta Biológica Colombiana 14:169-172.

Ramírez-Luna E., C. C. Castillo-Aguilar, E. Aceves-Navarro y E. Carrillo-Ávila (2005) Efecto de productos con reguladores de crecimiento sobre la floración y amarre de fruto en chile habanero. Revista Chapingo Serie Horticultura 11:93-98.

Ramírez H., C. Amado-Ramírez, A. Benavides-Mendoza. V. Robledo-Torres y A. Martínez-Osorio (2010) Prohexadiona-Ca, AG3, ANOXA y BA modifican indicadores fisiológicos y bioquímicos en chile Mirador. Revista Chapingo Serie Horticultura 16:83-89.
Ramírez H., B. Herrera-Gámez, Y. H. Méndez-Quiroz, A. Benavides-Mendoza, J. A. De La Cruz- Bretón, V. Álvarez-Mares. J. H. Rancaño-Arrioja y J. A. Villareal-Quintanilla (2008) Prohexadiona de calcio disminuye el contenido de giberelinas endógenas en ápices de tomate saladette y chile pimiento. Revista Chapingo Serie Horticultura 14:193-198.

Rodríguez J. L. (2002) Inducción a la floración y cuajado de frutos. Revista Productores de Hortalizas 24:20-23.

Uribe F. (2008) Chile habanero como opción de cultivo. Hortalizas. http://www.hortalizas.com/uncategorized/chile-habanerocomo-opcion-de-cultivo/ (Noviembre 2015) 